\title{
Emerging treatments in lung cancer - targeting the RLIP76 molecular transporter
}

\author{
This article was published in the following Dove Press journal: \\ Lung Cancer:Targets and Therapy \\ 4 November 2013 \\ Number of times this article has been viewed
}

\author{
Lawrence E Goldfinger ${ }^{1,2}$ \\ Seunghyung Lee' \\ 'Department of Anatomy and Cell \\ Biology, The Sol Sherry Thrombosis \\ Research Center, Temple University \\ School of Medicine, Philadelphia, PA, \\ USA; ${ }^{2}$ Cancer Biology Program, Fox \\ Chase Cancer Center, Philadelphia, \\ PA, USA
}

\begin{abstract}
Multidrug resistance in lung cancer cells is a significant obstacle in the treatment of lung cancer. Resistance to chemotherapeutic agents is often the result of efflux of the drugs from cancer cells, mediated by adenosine triphosphate (ATP)-dependent drug transport across the plasma membrane. Thus, identifying molecular targets in the cancer cell transport machinery could be a key factor in successful combinatorial therapy, along with chemotherapeutic drugs. The transport protein Ral-interacting protein of $76 \mathrm{kDa}$ (RLIP76), also known as Ralbinding protein 1 (RalBP1), is a highly promising target for lung cancer treatment. RLIP76 is an ATP-dependent non-ATP-binding cassette (ABC) transporter, responsible for the major transport function in many cells, including many cancer cell lines, causing efflux of glutathioneelectrophile conjugates of both endogenous metabolites and environmental toxins. RLIP76 is expressed in most human tissues, and is overexpressed in non-small-cell lung cancer cell lines and in many tumor types. The blockade of RLIP76 by various approaches has been shown to increase the sensitivity to radiation and chemotherapeutic drugs, and leads to apoptosis in cells. In xenograft tumor models in mice, RLIP76 blockade or depletion results in complete and sustained regression across many cancer cell types, including lung cancer cells. In addition to its transport function, RLIP76 has many other cellular and physiological functions based on its domain structure, which includes a unique Ral-binding domain and a Rho GTPase activating protein (RhoGAP)-catalytic domain as well as docking sites for multiple signaling proteins. As a Ral effector, RhoGAP, and adapter protein, RLIP76 has been shown to play important roles in endocytosis, mitochondrial fission, cell spreading and migration, actin dynamics during gastrulation, and Ras-induced tumorigenesis. Additionally, RLIP76 is also important for stromal cell function in tumors, as it was recently shown to be required for efficient endothelial cell function and angiogenesis in solid tumors. However, RLIP76 knockout mice are viable, and blockade effects appear to be selective for implanted tumors in mice, suggesting the possibility that RLIP76-targeting drugs may be successful in clinical trials. In this review, we outline the many cellular and physiological functions of RLIP76 in normal and cancer cells, and discuss the potential for RLIP76-based therapeutics in lung cancer treatment.
\end{abstract}

Keywords: RalBP1, tumor angiogenesis, ATP-dependent transport, chemotherapeutics, apoptosis, endothelial cell

\section{Introduction}

Lung cancer is the leading cause of cancer death for both men and women in the world, and every year 1.2 million new cases are diagnosed worldwide. ${ }^{1,2}$ In 2010, the most recent year for which detailed lung cancer statistics are available, patient survival was $20 \%-30 \%$. $^{2}$ Lung tumors have two major types: small-cell (SCLC) and non-small-cell (NSCLC) lung cancer. Roughly $80 \%-85 \%$ of lung cancers are NSCLC,
Correspondence: Lawrence E Goldfinger Temple University School of Medicine, 3420 N Broad Street, MRB 219 ,

Philadelphia, PA, 19140, USA

Tel +I 2157078157

Fax + I 2157076499

Email goldfinger@temple.edu submit your manuscript | www.dovepress.com

Dovepress

http://dx.doi.org// 0.2147/LCTT.S53672
Lung Cancer:Targets and Therapy 2013:4 6I-69

(c) (1) (5) 2013 Goldfinger and Lee. This work is published by Dove Medical Press Limited, and licensed under Creative Commons Attribution - Non Commercial (unported, v3.0) License. The full terms of the License are available at http://creativecommons.org/licenses/by-nc/3.0/. Non-commercial uses of the work are permitted without any further permission from Dove Medical Press Limited, provided the work is properly attributed. Permissions beyond the scope of the License are administered by Dove Medical Press Limited. Information on how to request permission may be found at: http://www.dovepress.com/permissions.php 
and metastatic disease at presentation is common in these patients. $^{3,4}$ Response rates to chemotherapeutic regimens are low; thus, lung cancer continues to be a major cause of cancer mortality. ${ }^{2,5}$

Selective and potent molecular targets for treating lung cancer are desperately needed. A promising approach is to prevent the ability of lung cancer cells to export chemotherapeutic drugs, trapping the drugs within the cancer cells and thereby making them more sensitive to drug cell-killing effects. Hence, this represents a combinatorial therapeutic approach including both the chemotherapeutic agent and transport blockers. A highly promising transport target is the protein Ral-interacting protein of $76 \mathrm{kDa}$ (RLIP76), an adenosine triphosphate (ATP)-dependent non-ATP-binding cassette $(\mathrm{ABC})$ transporter, which is responsible for the major transport function for the efflux of endogenous metabolites as well as chemotherapeutic agents in many cells and tissues. ${ }^{6-8}$ This review will focus on the mechanisms and regulatory effects of RLIP76 in cancer and its specific roles in lung cancer.

\section{Role of RLIP76 in cancer}

RLIP76 is expressed in most human tissues, including liver, heart, ovary, lung, muscle, and kidney as well in most human tumor cell lines, and is overexpressed in multiple cancers, such as lung and ovarian carcinomas and melanomas (Table 1). ${ }^{9-12}$ As a prominent cellular function of RLIP76 is the export of chemotherapy agents, it is a major factor in the mechanisms of drug resistance. Moreover, blockade of RLIP76 with targeting antibod-

Table I RLIP76 expression in normal tissues and over-expression in cancer cells

\begin{tabular}{|c|c|}
\hline & Reference \\
\hline \multicolumn{2}{|c|}{ Normal tissue } \\
\hline Erythrocytes & Sharma et $\mathrm{al}^{12,53}$ \\
\hline Heart & Awasthi et al'" \\
\hline Liver & Awasthi et al'" \\
\hline Lung & Singhal et $\mathrm{al}^{77,78}$ \\
\hline Kidney & Awasthi et al' \\
\hline Muscle & Awasthi et al"I \\
\hline Placenta & Awasthi et al" \\
\hline \multicolumn{2}{|c|}{ Cancer cell type } \\
\hline Melanoma & Lee et al, $^{69}$ Singhal et al $^{15}$ \\
\hline Colon & Singhal et $\mathrm{al}^{13}$ \\
\hline Kidney & Singhal et $\mathrm{al}^{67}$ \\
\hline Liver & Awasthi et al"I \\
\hline Lung & Singhal et al, ${ }^{13,35,66}$ Lee et $a^{69}$ \\
\hline Ovary & Awasthi et al" \\
\hline Prostate & Singhal et al $^{14}$ \\
\hline
\end{tabular}

Abbreviation: RLIP76, Ral-interacting protein of $76 \mathrm{kDa}$. ies or antisense has been shown to greatly increase the sensitivity to radiation and chemotherapy and to lead to pronounced tumor regression, in multiple types of solid tumors in mice, including xenografted tumors of lung cancer cells. ${ }^{13-15}$

\section{RLIP76 as a multifunctional protein}

Almost 20 years ago, RLIP76 was discovered by three different groups. ${ }^{16-18}$ The RALBP1 gene encoding RLIP76 is located on human chromosome $18 \mathrm{p} 11 .{ }^{6}$ RLIP76 is a modular, multifunctional protein (Table 2) of 655 amino acids, harboring an N-terminal putative helical domain of poorly characterized function, a central Rho GTPase activating protein (RhoGAP) domain, and a conserved Ral-binding domain (RalBD) near the C-terminus. The RalBD, which bears no homology to classical Ras-binding domains, supports interaction with activated RalA and RalB but not with Ras small G proteins. ${ }^{19}$ Like all Ras superfamily small G proteins, Ral proteins are signal transducers that become activated upon release of guanine diphosphate (GDP) and binds to guanine triphosphate (GTP), upon which Ral undergoes a conformational shift to expose high affinity binding sites for signaling effectors. Recently, Fenwick et al solved the nuclear magnetic resonance (NMR) solution structure of the

Table 2 Summary of RLIP76 cellular and physiological functions

\begin{tabular}{|c|c|}
\hline Function & Mechanism \\
\hline \multirow[t]{2}{*}{ GS-E transport } & ATPase activity, membrane translocation ${ }^{6,7,25,26}$ \\
\hline & PKC phosphorylation ${ }^{56}$ \\
\hline Spreading and & Interaction with R-Ras ${ }^{30}$ \\
\hline \multirow[t]{2}{*}{ migration } & Rac and Arf6 activation ${ }^{30}$ \\
\hline & Ral signaling ${ }^{43}$ \\
\hline Angiogenesis & Endothelial cell proliferation and migration \\
\hline \multirow[t]{3}{*}{ Actin remodeling } & Ral interaction ${ }^{24}$ \\
\hline & RhoGAP activity, regulation of Rac and \\
\hline & $\mathrm{Cdc} 42$ \\
\hline \multirow[t]{5}{*}{ Mitosis } & Shut-off of endocytosis through interaction \\
\hline & with POBI, REPSI, and CDKI ${ }^{38,39}$ \\
\hline & Centrosome/spindle and nuclear localization ${ }^{38,39}$ \\
\hline & Mitochondrial fission through RalA and \\
\hline & AuroraA ${ }^{29}$ \\
\hline \multirow[t]{2}{*}{ Apoptosis resistance } & Endo- and xenobiotics transport ${ }^{63-65}$ \\
\hline & Interaction with HSF-I ${ }^{16}$ \\
\hline \multirow[t]{2}{*}{ Endocytosis } & RalA signaling, binding $A P 2$ and $P O B I^{37}$ \\
\hline & Transport activity ${ }^{40}$ \\
\hline Heat shock response & Interaction with HSF-I ${ }^{41}$ \\
\hline
\end{tabular}

Notes: Cellular and physiological functions in normal and tumor tissue are shown in the left column, and cellular and signaling mechanisms are shown to the right. Abbreviations: ADP, adenosine diphosphate; AP2, activating protein 2; Arf6, ADP-ribosylation factor; ATP, adenosine triphosphate; Cdc42, cell division control protein 42; CDKI, cyclin-dependent kinase I; GS-E, glutathione-electrophile conjugate; GTP; guanosine triphosphate; HSF-I, heat shock factor I; PKC, protein kinase C; POBI, partner of RaIBPI; REPSI, RaIBPI-associated Eps domain-containing protein I; RhoGAP, Rho GTPase activating protein; RLIP76, Ral-interacting protein of 76 kDa. 
RalBD of RLIP76 in complex with RalB, which revealed a novel binding face consisting of a coiled coil in the RalBD that binds to both of the so-called switch regions of Ral that "switch" conformation upon GTP binding. ${ }^{20,21}$ They further found that RLIP76 can compete with other Ral effectors, such as the exocytic modulators Sec5 and Exo84. ${ }^{21}$ Activated Ral recruits RLIP76 from the cytosol to the plasma membrane, suggesting that a key role of this regulatory step is in directing the localization of RLIP76 to subcellular locales for signal propagation. ${ }^{22}$ Thus, RLIP76 is a unique Ral effector, connecting upstream activation of Ral to downstream molecular and cellular events.

Functionally, the Ral-effector property of RLIP76 was originally described as linking Ral to Rho GTPase pathways through the RhoGAP domain. ${ }^{6,17,18}$ The Rho subfamily of Ras small G proteins, most prominently RhoA, Rac, and cell division control protein (Cdc)42, are, like Ras and Ral, regulated by guanine nucleotide exchange, such that RhoGAPs facilitate conversion from the GTP-bound active state to the GDP-bound inactive state. Signaling by these small $\mathrm{G}$ proteins leads to actin remodeling and altered cell morphologies, with Rac being associated with the formation of broad lamellipodia protrusions and $\mathrm{Cdc} 42$ with filopodia spikes. ${ }^{23}$ Early studies showed that RLIP76 supports RhoGAP activity towards $\mathrm{Rac}$ and $\mathrm{Cdc} 42$, but not RhoA, in vitro. Since Ral can be activated downstream of Ras, RLIP76 was proposed to bridge Ras activation - typically associated with mitogen signaling - with the activation of Rho proteins and cytoskeletal remodeling, via RLIP76 interaction with GTP-Ral. This cellular function was later shown to relate to physiological effects, as RLIP76 interaction with RalB modulates actin remodeling during gastrulation in Xenopus oocytes. ${ }^{24}$ Thus, an important cellular and physiological function of RLIP76 is to couple Ral-effector function with Rho signaling and actin cytoskeletal remodeling, promoting altered cell morphologies.

Since its initial characterization as a Ral effector and RhoGAP, RLIP76 has been implicated in various other cellular functions at different locations within cells, all of which are likely contributors to its efficacy as a putative cancer therapy target (Table 2). RLIP76 also functions as an ATP-dependent glutathione-conjugate transporter for small molecules, ${ }^{6}$ including anticancer drugs and endogenous metabolites, ${ }^{7,25,26}$ and in endocytosis, ${ }^{27,28}$ mitochondrial fission, ${ }^{29}$ cell spreading and migration, ${ }^{29}$ and Ras-induced tumorigenesis (although RLIP76 is not critical for Ras/Ral-mediated tumorigenesis, indicating RLIP76 is not the major effector in this process). ${ }^{31,32}$
RLIP76 also contains many putative sites of protein phosphorylation by several kinases, such as protein kinase $\mathrm{C}$ (PKC)s (a family of serine/threonine [Ser/Thr] kinases) as well as Ral interacting kinase, and many of the utilized sites in cells are Ser and Thr residues in the N-terminal domain. ${ }^{33,34}$ PKC-dependent RLIP76 phosphorylation appears to play an important role in its functions in lung cancer (described below). ${ }^{35,36}$

An important but possibly underappreciated function of RLIP76 is as an adapter molecule. Identified binding partners (besides RalA and RalB) include activating protein (AP)2, partner of RalBP1 (POB1), heat shock factor (HSF)-1, R-Ras, and RalBP1-associated Eps domain-containing protein (Reps1). Through these interactions, RLIP76 participates in various cellular functions, by coupling related molecules in signaling pathways. For example, in addition to linking Ral to Rho pathways, RLIP76 also regulates Ral signaling in the regulation of endocytic recycling of many proteins, including growth factor receptors, through binding to AP2 and POB $1 .{ }^{37}$ During mitosis, RLIP76 is phosphorylated, redistributes to centrosomes and the mitotic spindle, and interacts with POB1 and mitotic kinase cyclin-dependent kinase (CDK) 1, leading to Ral-dependent shut off of endocytosis during mitosis. ${ }^{38,39}$ Nuclear localization of RLIP76 is mediated by nuclear localization sequences in the N-terminal domain, whereas putative coiled coil domains in the C-terminus adjacent to the RalBD are necessary for retention in the nucleus. ${ }^{39}$ Additionally, during mitosis, RLIP76 also localizes to the mitochondria where it couples Ral phosphorylation by mitotic kinase Aurora A to signaling necessary for mitochondrial fission and proper distribution in the daughter cells. ${ }^{29}$ Furthermore, the role of RLIP76 in endocytosis may be coupled to its transport activity. ${ }^{40}$ RLIP76 also appears to regulate stress-induced transcriptional activation by forming complexes with HSF-1. ${ }^{41}$ Finally, our group identified RLIP76 as a selective effector of the small GTPase R-Ras and found that RLIP76 regulates R-Ras signaling, leading to cell spreading and migration..$^{30,42}$ These effects are the result of RLIP76 adapter function, whereby it recruits ARNO, a small GTPase guanine exchange factor, to R-Ras at recycling endosomes, to regulate vesicular trafficking necessary for spreading and migration ${ }^{29}$ (our unpublished results support this). RLIP76 also potentiates Ral-mediated cell spreading, potentially through similar signaling pathways. ${ }^{43}$ Thus, RLIP76 regulates a broad spectrum of molecular, cellular, and physiological processes, many of which stem from its function as a molecular adapter in various cellular locales. 


\section{RLIP76 molecular transport function in cancer}

Of the many functions of RLIP76 related to cancer initiation and progression, the most thoroughly characterized is as a molecular transporter of glutathione-electrophile conjugates (GS-E). GS-Es form by thioether conjugation of glutathione (GSH) (between 1-10 mM GSH cytosolic concentration in cells), an electron donor and thus a reducing agent, with electrophilic or oxidant chemicals that are derived both endogenously (endobiotics) and from the environment (xenobiotics). In this way, GSH acts as a scavenger for alkylating agents and other electrophiles. ${ }^{44}$ GS-E conjugates become trapped in cells and require energy-dependent transport for their removal, to prevent toxicity caused both by excessive GS-E and impairment of the overall process of reduction of electrophilic toxins. ${ }^{45,46}$ Some of this transport function is carried out by $\mathrm{ABC}$ transporters. ${ }^{47,48}$ Thus, in addition to the removal from cells of toxic endobiotics, such as 4-hydroxynonenal (4-HNE), GS-E transport is also essential for protection from xenobiotics. ${ }^{45,49}$ Multidrug resistance (MDR), particularly for alkylating chemotherapeutic drugs, is very often the result of a failure of transport in the target cells; hence, transporters such as the ABC type are classified as MDR proteins, which have long been pursued as therapeutic targets to inhibit drug resistance in cancer cells. ${ }^{50,51}$

However, despite a great deal of attention to the targeting of transporters in cancer therapy, early attempts have not been successful. One reason for this may be that MDR and associated proteins account for only a fraction of the transport activity in many cells; instead, about two-thirds of cellular transport activity appears to be the work of RLIP76. ${ }^{10,52,53}$ In the early 1980s, Awasthi et al discovered a 1-chloro-2,4-dinitrobenzene (DNP-SG) ATPase in erythrocytes, which acts as the primary ATP-dependent transporter of the DNP-SG GSH conjugate..$^{54,55}$ The same group later found that this transporter is identical to RLIP76. RLIP76 is a novel, non-ABC type transporter, which utilizes both of its two ATP binding sites - in the N-terminal domain (aa 69-74) and adjacent to the RalBD (418-425) - for ATPase and transport activity but which lacks the canonical Walker domain of ABC-type transporters..$^{25}$ Interestingly, PKC-mediated phosphorylation of RLIP76 increases its transport activity. ${ }^{56}$ A wealth of subsequent studies have shown that RLIP76 is the major transporter for a wide range of structurally distinct endobiotics and xenobiotic chemotherapy agents, including GS-E, doxorubicin, sulfates, leukotriene C4, vinorelbine, glucorinides, colchicine, and other organic anions and cations. ${ }^{11,57-62}$ Due to this broad spectrum of transport targets,
RLIP76 plays important roles in resistance to apoptosis due to heat shock and oxidative stress (in part, through interaction with HSF-1), radiation sensitivity, and perhaps most prominently, to MDR in cancer therapy. ${ }^{63-65}$

The various cellular functions of RLIP76, and in particularly its ATPase transport activity, have been shown to translate directly to MDR in cancer cells in many types of tumors. The blockade of RLIP76 by antisense or short inhibitory ribonucleic acid (siRNA) depletion or with antiRLIP76 antibodies (presumed to block membrane-targeted RLIP76) has been shown to cause apoptosis in SCLC and NSCLC, leukemia, lymphoma, melanoma, colon cancer, and prostate cancer cell lines, and RLIP76 blockade or depletion synergizes with chemotherapeutic agents, such as anthracyclines and Vinca alkaloids (eg, vinorelbine) to further enhance apoptosis in these cancer cell lines. These in vitro effects have translated, in every case, to pronounced in vivo effects in tumor xenografts: the blockade of RLIP76 has led to regression of tumors formed by xenografted lung cancer, melanoma, colon cancer, prostate cancer, and kidney cancer cells in mice. ${ }^{13-15,60,66,67}$ In at least some cases, like in prostate cancer cells, anti-RLIP76 antibodies were equally as effective as siRNA, suggesting that the transport function of RLIP76 is the major driver of MDR in these cells. ${ }^{14}$ Conversely, the ectopic administration of RLIP76, such as by transfection in cancer cell lines or with proteoliposomes in vivo, has been shown to restore transport and the efflux of xenobiotics, and enhance MDR in the tumors, reversing the regressive effects of RLIP76 blockade. ${ }^{52,60,66}$

\section{RLIP76 in tumor angiogenesis}

In addition to its broad variety of functions in normal epithelial and erythroid cells and in transformed cells in tumors, RLIP76 also has important roles in stromal cells, with consequences for solid tumor progression, survival, and possibly metastasis. In particular, RLIP76 has unique functions in endothelial cells, specialized squamous epithelia that form the inner core of all blood vessels and are the sole cellular components of the microvasculature (capillaries). ${ }^{68,69} \mathrm{We}$ have recently found a role for RLIP76 in tumor angiogenesis. Angiogenesis, the outgrowth of new blood vessels from existing ones, is required for the progression of tumor growth and metastasis. Solid tumors require a nutrient blood supply to grow beyond $\sim 1 \mathrm{~mm}$ diameter, and the inhibition of tumor angiogenesis has long been pursued as an approach to preventing tumor growth and subsequent metastasis. ${ }^{70}$ Angiogenesis is a complex process resulting from combined simultaneous upregulation of proliferation and migration in 
endothelial cells. During the initial stages of angiogenesis, proliferating and migrating endothelial cells convert to a spindle-shaped morphology and organize into branched capillary networks, which differentiate into fully-formed lumenal vessels carrying blood from the source vasculature to the new sites, such as into solid tumors. ${ }^{70}$

Based on the ability of RLIP76 to interact with R-Ras a modulator of tumor angiogenesis - as well as with other proteins important for endothelial function (described below), we recently investigated a potential physiological role for RLIP76 in angiogenesis, in solid tumors xenografted in mice. Tumor growth from the B16 melanoma or Lewis lung carcinoma cells xenografted into the flanks of $\mathrm{C} 57 \mathrm{Bl} / 6$ wild type (WT) mice was blunted in isogenic RLIP76 ${ }^{-/-}$mice. We used three-dimensional (3-D) X-ray microcomputed tomography to reconstruct the tumor vascular structures in resected tumors from WT and RLIP76 ${ }^{-/-}$mice and found defects in both the extent and form of tumor angiogenesis in the RLIP76 ${ }^{-/}$mice. Specifically, the tumor vascular volumes were diminished, and the vessels were fewer in number, shorter, and narrower in RLIP76 ${ }^{-/-}$mice than in WT mice. Moreover, we found that angiogenesis in basement membrane matrix plugs was blunted in the knockout mice in the absence of tumor cells, with endothelial cells isolated from the lungs of these animals exhibiting defects in migration, proliferation, and cord formation in vitro. ${ }^{69}$ Furthermore, in a model of induced skin carcinogenesis, to which RLIP76 ${ }^{-1}$ mice are already resistant,${ }^{40}$ the tumors that did form showed diminished angiogenesis; conversely, liposome-mediated reconstitution of RLIP76 expression restored tumor growth and tumor angiogenesis in this model. ${ }^{69}$ Thus, in addition to regulating tumor cell growth, RLIP76 is required for efficient endothelial cell function and angiogenesis in solid tumors.

Importantly, when we xenografted tumor cells in which RLIP76 expression had been stably suppressed with a short hairpin ribonucleic acid (shRNA), tumor growth was slightly but significantly reduced in both the WT and RLIP76 $6^{-/}$mice compared with tumors expressing control shRNA. ${ }^{69}$ This result points to a dual physiological role for RLIP76 in tumor progression: RLIP76 regulates tumor progression by modulating survival of the tumor cells themselves, as previously demonstrated, as well as by supporting neovascularization derived from the host vascular cells. Thus, RLIP76 contributes independently to the growth of tumor cells and to angiogenesis from the host vasculature in solid tumors, such that combined depletion in both compartments further reduces tumor growth and tumor angiogenesis over those observed by knockout in the host animal only. These results strengthen the case for RLIP76 as a potent molecular target in cancer, as blocking RLIP76 appears to diminish tumor growth by inhibiting both tumor cell growth and the angiogenesis required for tumor expansion.

\section{Molecular and cellular mechanisms of RLIP76 in tumor angiogenesis}

The physiological effects of RLIP76 depletion or blockade in tumor angiogenesis are likely to be manifold, reflecting the multifunctional nature of RLIP76 in cells. A novel cellular function of RLIP76, which may be unique to endothelial cells, is in cell survival in cases of oxidative stress. Based on a complementary deoxyribonucleic acid (cDNA) library screen of microvascular endothelial cells with patient sera, Margutti et al found that patients with several diseases resulting from endothelial dysfunction expressed autoantibodies to RLIP76. ${ }^{68}$ In vitro, both macro- and microvascular endothelial cells were shown to respond to oxidative stress by redistributing RLIP76 to the plasma membrane, and the treatment of these cells with RLIP76 autoantibodies led to increased intracellular 4-HNE and phosphorylation of Jun-kinase, decreased intracellular GSH, and caused caspase-dependent apoptosis. ${ }^{68}$ Thus, endothelial dysfunction by RLIP76 antibodies may relate to its roles as a transporter. The endothelium is uniquely sensitive to oxidative stress, which can cause endothelium-induced development of atherosclerosis, endothelial permeability, expression of adhesion molecules (which can capture leukocytes and promote inflammatory responses), as well as to apoptosis leading to breakdown of the vascular wall. ${ }^{71}$ Interestingly, the endothelial apoptosis caused by RLIP76 antibodies appears to be significantly more pronounced in cells derived from women than from men. ${ }^{72}$ Potential explanations for this gender disparity in RLIP76 sensitivity are the apparently higher surface expression of RLIP76 in endothelial cells derived from women than from men and the finding that RLIP76 sensitivity increased in cells derived from women, but not from men, following treatment with estrogen. ${ }^{72}$ Thus, one mechanism of blockade of tumor angiogenesis by targeting RLIP76 such as via antibodies, may be by induction of endothelial apoptosis.

Besides regulating oxidative stress-induced apoptosis, RLIP76 also has other molecular and cellular functions that may contribute to its roles in angiogenesis. As an adapter, RLIP76 interacts with multiple proteins that regulate cell morphology, intracellular vesicular trafficking, and downstream effects on cell adhesion and migration - all of which are important processes in endothelial cell-mediated angiogenesis. The central RhoGAP domain supports GAP activity towards 
small GTPases Cdc42 and Rac1 in vitro, leading to inactivation of these proteins but not to RhoA, as outlined above. However, in cells, RLIP76 expression is required for adhesion-induced activation of Rac1, leading to remodeling of the actin cytoskeleton, cell spreading, and cell migration. ${ }^{30}$ These molecular and cellular functions of RLIP76 relate to its ability to interact selectively with the activated (GTP-bound) form of the Ras family small GTPase R-Ras, which is an essential modulator of endothelial barrier function and angiogenesis. ${ }^{30,73-76}$ RLIP76 expression is required for R-Ras to promote Rac activation and Rac-dependent cell spreading and migration; hence, RLIP76 may be an important mediator in the endothelial functions of R-Ras. ${ }^{30}$ In addition, RLIP76 interacts with a guanine exchange factor for the small $G$ protein ADP-ribosylation factor (Arf)6, a key driver of vesicular trafficking and upstream of Rac activation. ${ }^{30}$ Furthermore, as an effector of activated Ral GTPase, RLIP76 regulates the exocytic pathway and actin remodeling downstream of Ral, as outlined above. ${ }^{24}$ A newly described function of the RLIP76/ Ral complex is in recruiting proteins essential for mitochondrial fission during mitosis. ${ }^{29}$ Thus, the adapter function of RLIP76, through its ability to coordinate signaling through a variety of small $\mathrm{G}$ protein signaling networks, plays important roles in vesicular trafficking, mitochondrial dynamics, actin remodeling, cell morphology, and motility - all of which are likely to contribute to the requirement for RLIP76 expression in endothelial function and angiogenesis in tumors.

\section{Specific role of RLIP76 in lung cancer}

The role of RLIP76 as a transporter mediating MDR has been most thoroughly studied in the context of lung cancer. Early studies in this area investigated 13 native human lung cancer cell lines and found that RLIP76 purified from NSCLC had about twofold higher ATPase activity than that from SCLC cell lines, perhaps due to different posttranslational modifications, and RLIP76-mediated transport of doxorubicin was similarly enhanced in NSCLC compared with SCLC. ${ }^{77,78}$ A recent study found RLIP76 is overexpressed in multiple NSCLC cell lines. ${ }^{79}$ Another early study found that blockade of RLIP76 with specific antibodies synergized with doxorubicin to cause apoptosis in NSCLC. ${ }^{80} \mathrm{~A}$ subsequent study showed that knockdown of RLIP76 by siRNA caused apoptosis in six different NSCLC cell lines, whereas augmenting RLIP76 levels led to MDR and the prevention of apoptosis by both endobiotics (4-HNE) and doxorubicin. ${ }^{66}$ Further support for RLIP76 in mediating chemotherapy resistance in lung cancer came with the finding that depletion or aug- mentation of RLIP76 had the same corresponding effects in both NSCLC and SCLC cells with respect to vinorelbine, a Vinca alkaloid with apparently less resistance in NSCLC than similar drugs. ${ }^{52} \mathrm{HSF}-1$ and POB1, binding partners of RLIP76, appear to be inhibitors of RLIP76 transport function, at least in NSCLC cells, which may explain why these proteins are associated with drug sensitivity. ${ }^{63,81}$ However, the most direct evidence to date for a prominent role of RLIP76 in lung cancer, and its putative efficacy as a therapeutic target, was the finding that depletion of RLIP76 either with antisense or with anti-RLIP76 antibodies caused rapid and complete regression, and long-term remission of tumors in mice xenografted with two different NSCLC cell lines. Antibody and antisense treatment yielded similar results, pointing to the importance of the transport function in the regression, and furthermore, RLIP76 blockade enhanced the regressive effects of vinorelbine in this model. ${ }^{13}$ Thus, RLIP76 is the principal mediator of ATP-dependent transport, driving efflux of chemotherapeutic agents in lung cancer cells and tumors and leading to MDR, making RLIP76 a prominent target in developing chemotherapeutic approaches to fight lung cancer.

\section{Critical analysis of the potential for targeting RLIP76 in lung cancer management}

RLIP76 is a potent antiapoptotic protein, due largely to its roles as a molecular transporter. Its efficacy as a cancer therapeutic target - having been demonstrated in various models of tumor regression in mice - is also based on its apparent selectivity for cancer cells in these functions (Figure 1). ${ }^{9}$ The upregulation of RLIP76 expression in multiple tumor types and cell lines supports this possibility. Moreover, based on its role in tumor angiogenesis, it is likely that regressive effects of RLIP76 targeting in tumor xenografts are the result of combinatorial effects in tumor cells and stromal cells (Figure 1). The prevention of xenografted tumor growth and synergy with the inhibition of tumor angiogenesis in RLIP76 knockout mice and conversely, the enhanced tumor angiogenesis observed following ectopic application of RLIP76 in the knockout mice, ${ }^{69}$ strongly supports the idea of a one-two punch attack on the growth of solid tumors, by blocking RLIP76 function in tumor cells and the tumor vasculature. However, it must be noted that the in vivo studies to date have focused on xenografted tumors in mouse flanks, and direct application to cancer in the lung has yet to be determined. Furthermore, besides focused evaluation of the xenografted tumors in control mice following RLIP76 blockade or global depletion, it remains to 


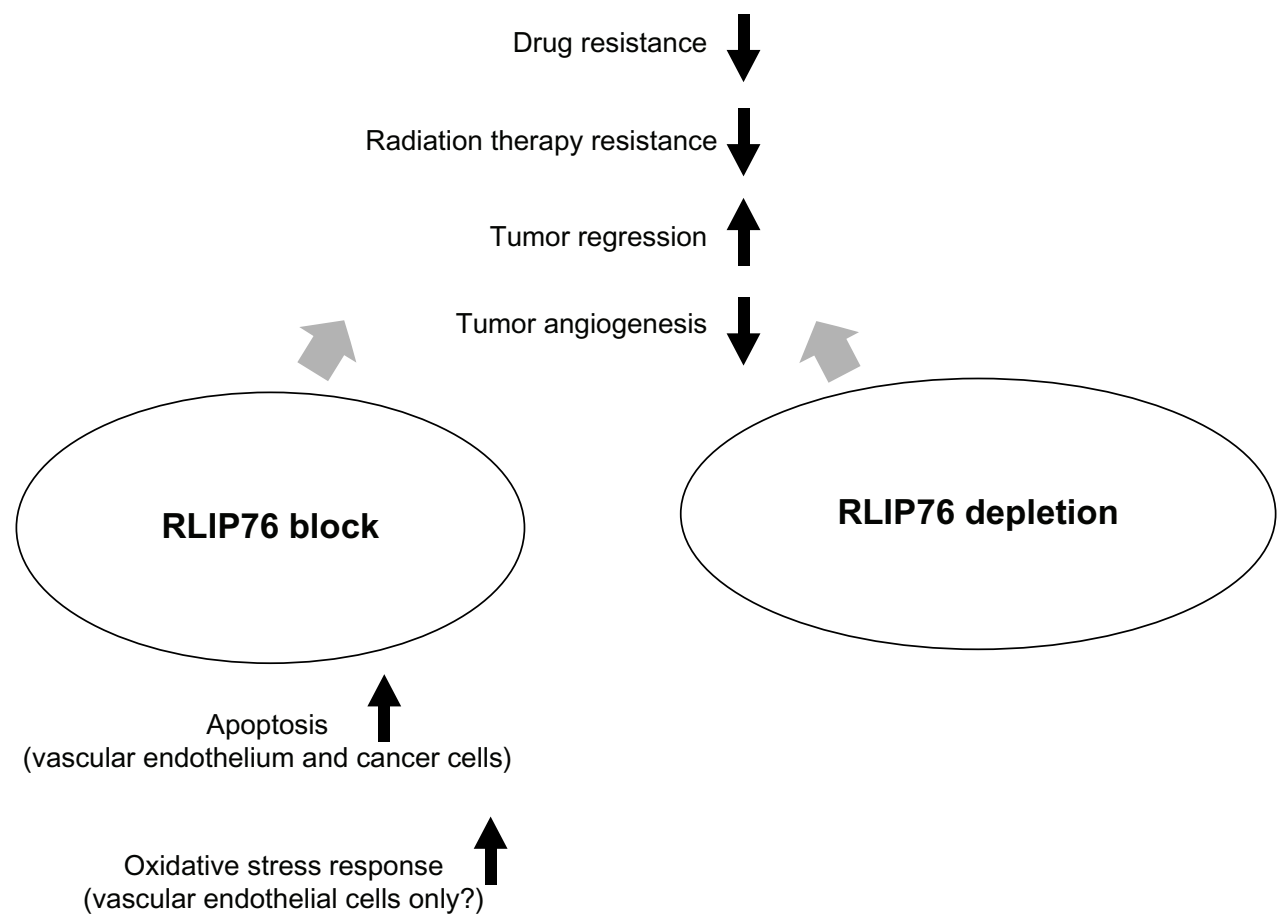

Figure I RLIP76 blockade in tumorigenesis and tumor progression.

Notes: Effects of RLIP76 blockade in tumors are shown. Effects including inhibition of tumor angiogenesis and heat shock responses are likely manifested in tumor cells and tumor stromal cells.

Abbreviation: RLIP76, Ral-interacting protein of $76 \mathrm{kDa}$.

be seen whether these treatments have other adverse vascular effects. Indeed, the role of RLIP76 in the oxidative stress response and apoptosis in endothelial cells, coupled with elevated levels of RLIP76 autoantibodies in Behçet's disease, systemic lupus, and other vascular pathologies, ${ }^{68}$ suggest that more investigation in this area is needed. On the other hand, RLIP76 is not essential for development in mice, which may reflect the particular effects of RLIP76 blockade in tumors. Recently, yet another pathogenic function of RLIP76 was identified, in metabolic disease, ${ }^{82}$ hence, as with any putative therapeutic, caution will be required in the path towards targeting RLIP76 in lung cancer and other cancers. However, the wealth of preliminary evidence points strongly towards pursuing this goal of developing RLIP76-targeting therapeutic approaches in the treatment of lung cancer.

\section{Conclusion}

In the ongoing search for molecular targets in lung cancer treatment, RLIP76 is among the most promising candidates. Its multifactorial contributions to tumor growth and survival are reflected in the complete regression of lung cancer cell tumors by blocking or depleting RLIP76 in mice. Most likely, drugs targeting RLIP76 will be most useful in combinatorial therapies, such as with classical chemotherapeutic agents, such as doxorubicin. However, off-target effects in other tissues will have to be closely monitored, and it remains to be seen whether the animal studies to date will translate into success in clinical trials. A more thorough understanding of the molecular mechanisms of RLIP76 function could be the key to developing selective therapeutic approaches in targeting RLIP76, in the treatment of lung cancer.

\section{Acknowledgments}

This work was supported by NIH grant HL093416 to LEG and by the American Heart Association Postdoctoral Fellowship 12POST12040257 to SL.

\section{Disclosure}

The authors report no conflicts of interest in this work.

\section{References}

1. Greenlee RT, Murray T, Bolden S, Wingo PA. Cancer statistics, 2000. CA Cancer J Clin. 2000;50(1):7-33.

2. Jemal A, Siegel R, Xu J, Ward E. Cancer statistics, 2010. CA Cancer J Clin. 2010;60(5):277-300.

3. Goldstraw P, Crowley J, Chansky K, et al; International Association for the Study of Lung Cancer International Staging Committee; Participating Institutions. The IASLC Lung Cancer Staging Project: proposals for the revision of the TNM stage groupings in the forthcoming (seventh) edition of the TNM Classification of malignant tumours. J Thorac Oncol. 2007;2(8):706-714.

4. Riaz SP, Lüchtenborg M, Coupland VH, Spicer J, Peake MD, Møller H. Trends in incidence of small cell lung cancer and all lung cancer. Lung Cancer. 2012;75(3):280-284. 
5. Schiller JH, Harrington D, Belani CP, et al; Eastern Cooperative Oncology Group. Comparison of four chemotherapy regimens for advanced nonsmall-cell lung cancer. $N$ Engl J Med. 2002;346(2):92-98.

6. Awasthi S, Cheng J, Singhal SS, et al. Novel function of human RLIP76: ATP-dependent transport of glutathione conjugates and doxorubicin. Biochemistry. 2000;39(31):9327-9334.

7. Awasthi S, Singhal SS, Sharma R, Zimniak P, Awasthi YC. Transport of glutathione conjugates and chemotherapeutic drugs by RLIP76 (RALBP1): a novel link between G-protein and tyrosine kinase signaling and drug resistance. Int J Cancer. 2003;106(5):635-646.

8. Vatsyayan R, Lelsani PC, Awasthi S, Singhal SS. RLIP76: a versatile transporter and an emerging target for cancer therapy. Biochem Pharmacol. 2010;79(12):1699-1705.

9. Awasthi S, Singhal SS, Awasthi YC, et al. RLIP76 and Cancer. Clin Cancer Res. 2008;14(14):4372-4377.

10. Awasthi S, Singhal SS, Srivastava SK, et al. Adenosine triphosphatedependent transport of doxorubicin, daunomycin, and vinblastine in human tissues by a mechanism distinct from the P-glycoprotein. J Clin Invest. 1994;93(3):958-965.

11. Awasthi YC, Singhal SS, Gupta S, et al. Purification and characterization of an ATPase from human liver which catalyzes ATP hydrolysis in the presence of the conjugates of bilirubin bile acids and glutathione. Biochem Biophys Res Commun. 1991;175(3):1090-1096.

12. Sharma R, Gupta S, Singh SV, et al. Purification and characterization of dinitrophenylglutathione ATPase of human erythrocytes and its expression in other tissues. Biochem Biophys Res Commun. 1990;171(1): 155-161.

13. Singhal SS, Singhal J, Yadav S, et al. Regression of lung and colon cancer xenografts by depleting or inhibiting RLIP76 (Ral-binding protein 1). Cancer Res. 2007;67(9):4382-4389.

14. Singhal SS, Roth C, Leake K, Singhal J, Yadav S, Awasthi S. Regression of prostate cancer xenografts by RLIP76 depletion. Biochem Pharmacol. 2009;77(6):1074-1083.

15. Singhal SS, Awasthi YC, Awasthi S. Regression of melanoma in a murine model by RLIP76 depletion. Cancer Res. 2006;66(4):2354-2360.

16. Cantor SB, Urano T, Feig LA. Identification and characterization of Ral-binding protein 1, a potential downstream target of Ral GTPases. Mol Cell Biol. 1995;15(8):4578-4584.

17. Jullien-Flores V, Dorseuil O, Romero F, et al. Bridging Ral GTPase to Rho pathways. RLIP76, a Ral effector with CDC42/Rac GTPaseactivating protein activity. J Biol Chem. 1995;270(38):22473-22477.

18. Park SH, Weinberg RA. A putative effector of Ral has homology to Rho/ Rac GTPase activating proteins. Oncogene. 1995;11(11):2349-2355.

19. Bauer B, Mirey G, Vetter IR, et al. Effector recognition by the small GTP-binding proteins Ras and Ral. J Biol Chem. 1999;274(25): 17763-17770.

20. Mott HR, Owen D. RLIP76 (RalBP1): The first piece of the structural puzzle. Small GTPases. 2010;1(3):157-160.

21. Fenwick RB, Campbell LJ, Rajasekar K, et al. The RalB-RLIP76 complex reveals a novel mode of ral-effector interaction. Structure. 2010;18(8):985-995.

22. Matsubara K, Hinoi T, Koyama S, Kikuchi A. The post-translational modifications of Ral and Rac1 are important for the action of Ralbinding protein 1, a putative effector protein of Ral. FEBS Lett. 1997; 410(2-3):169-174.

23. Burridge K. Crosstalk between Rac and Rho. Science. 1999;283(5410): 2028-2029.

24. Lebreton S, Boissel L, Iouzalen N, Moreau J. RLIP mediates downstream signalling from RalB to the actin cytoskeleton during Xenopus early development. Mech Dev. 2004;121(12):1481-1494.

25. Awasthi S, Cheng JZ, Singhal SS, et al. Functional reassembly of ATPdependent xenobiotic transport by the $\mathrm{N}$ - and $\mathrm{C}$-terminal domains of RLIP76 and identification of ATP binding sequences. Biochemistry. 2001;40(13):4159-4168.

26. Awasthi S, Sharma R, Yang Y, et al. Transport functions and physiological significance of $76 \mathrm{kDa}$ Ral-binding GTPase activating protein (RLIP76). Acta Biochim Pol. 2002;49(4):855-867.
27. Nakashima S, Morinaka K, Koyama S, et al. Small G protein Ral and its downstream molecules regulate endocytosis of EGF and insulin receptors. EMBO J. 1999;18(13):3629-3642.

28. Rossé C, L'Hoste S, Offner N, Picard A, Camonis J. RLIP, an effector of the Ral GTPases, is a platform for Cdk1 to phosphorylate epsin during the switch off of endocytosis in mitosis. J Biol Chem. 2003;278(33): 30597-30604

29. Kashatus DF, Lim KH, Brady DC, Pershing NL, Cox AD, Counter CM. RALA and RALBP1 regulate mitochondrial fission at mitosis. Nat Cell Biol. 2011;13(9):1108-1115.

30. Goldfinger LE, Ptak C, Jeffery ED, Shabanowitz J, Hunt DF, Ginsberg MH. RLIP76 (RalBP1) is an R-Ras effector that mediates adhesion-dependent Rac activation and cell migration. $J$ Cell Biol. 2006;174(6):877-888.

31. Lim KH, Baines AT, Fiordalisi JJ, et al. Activation of RalA is critical for Ras-induced tumorigenesis of human cells. Cancer Cell. 2005;7(6):533-545.

32. Issaq SH, Lim KH, Counter CM. Sec5 and Exo84 foster oncogenic ras-mediated tumorigenesis. Mol Cancer Res. 2010;8(2):223-231.

33. Herlevsen MC, Theodorescu D. Mass spectroscopic phosphoprotein mapping of Ral binding protein 1 (RalBP1/Rip1/RLIP76). Biochem Biophys Res Commun. 2007;362(1):56-62.

34. Jilkina O, Bhullar RP. A serine kinase associates with the RAL GTPase and phosphorylates RAL-interacting protein 1. Biochim Biophys Acta. 2006;1763(9):948-957.

35. Singhal SS, Yadav S, Singhal J, Drake K, Awasthi YC, Awasthi S. The role of PKCalpha and RLIP76 in transport-mediated doxorubicinresistance in lung cancer. FEBS Lett. 2005;579(21):4635-4641.

36. Singhal SS, Yadav S, Singhal J, Awasthi YC, Awasthi S. Mitogenic and drug-resistance mediating effects of PKCalpha require RLIP76. Biochem Biophys Res Commun. 2006;348(2):722-727.

37. Jullien-Flores V, Mahé Y, Mirey G, et al. RLIP76, an effector of the GTPase Ral, interacts with the AP2 complex: involvement of the Ral pathway in receptor endocytosis. J Cell Sci. 2000;113(Pt 16): 2837-2844.

38. Kariya K, Koyama S, Nakashima S, Oshiro T, Morinaka K, Kikuchi A. Regulation of complex formation of POB1/epsin/adaptor protein complex 2 by mitotic phosphorylation. J Biol Chem. 2000;275(24): 18399-18406.

39. Fillatre J, Delacour D, Van Hove L, et al. Dynamics of the subcellular localization of RalBP1/RLIP through the cell cycle: the role of targeting signals and of protein-protein interactions. FASEB J. 2012;26(5): 2164-2174.

40. Singhal SS, Wickramarachchi D, Yadav S, et al. Glutathione-conjugate transport by RLIP76 is required for clathrin-dependent endocytosis and chemical carcinogenesis. Mol Cancer Ther. 2011;10(1):16-28.

41. Hu Y, Mivechi NF. HSF-1 interacts with Ral-binding protein 1 in a stress-responsive, multiprotein complex with HSP90 in vivo. $J$ Biol Chem. 2003;278(19):17299-17306.

42. Goldfinger LE, Ptak C, Jeffery ED, et al. An experimentally derived database of candidate Ras-interacting proteins. J Proteome Res. 2007; 6(5):1806-1811.

43. Takaya A, Ohba Y, Kurokawa K, Matsuda M. RalA activation at nascent lamellipodia of epidermal growth factor-stimulated Cos7 cells and migrating Madin-Darby canine kidney cells. Mol Biol Cell. 2004;15(6): 2549-2557.

44. Awasthi YC, Chaudhary P, Vatsyayan R, Sharma A, Awasthi S, Sharma R. Physiological and pharmacological significance of glutathione-conjugate transport. J Toxicol Environ Health B Crit Rev. 2009; 12(7):540-551.

45. Cheng JZ, Sharma R, Yang Y, et al. Accelerated metabolism and exclusion of 4-hydroxynonenal through induction of RLIP76 and hGST5.8 is an early adaptive response of cells to heat and oxidative stress. $J$ Biol Chem. 2001;276(44):41213-41223.

46. Awasthi YC, Ansari GA, Awasthi S. Regulation of 4-hydroxynonenal mediated signaling by glutathione S-transferases. Methods Enzymol. 2005;401:379-407.

47. Ishikawa T. The ATP-dependent glutathione S-conjugate export pump. Trends Biochem Sci. 1992;17(11):463-468. 
48. Jedlitschky G, Leier I, Buchholz U, Barnouin K, Kurz G, Keppler D. Transport of glutathione, glucuronate, and sulfate conjugates by the MRP gene-encoded conjugate export pump. Cancer Res. 1996;56(5): 988-994.

49. Srivastava SK, Hu X, Xia H, et al. ATP-dependent transport of glutathione conjugate of 7beta, 8alpha-dihydroxy-9alpha,10alpha-oxy7,8,9,10-tetrahydrobenzo[a]pyrene in murine hepatic canalicular plasma membrane vesicles. Biochem J. 1998;332(Pt 3):799-805.

50. Ambudkar SV, Dey S, Hrycyna CA, Ramachandra M, Pastan I, Gottesman MM. Biochemical, cellular, and pharmacological aspects of the multidrug transporter. Annu Rev Pharmacol Toxicol. 1999;39: 361-398.

51. Borst P, Evers R, Kool M, Wijnholds J. A family of drug transporters: the multidrug resistance-associated proteins. J Natl Cancer Inst. 2000; 92(16):1295-1302.

52. Stuckler D, Singhal J, Singhal SS, Yadav S, Awasthi YC, Awasthi S. RLIP76 transports vinorelbine and mediates drug resistance in nonsmall cell lung cancer. Cancer Res. 2005;65(3):991-998.

53. Sharma R, Singhal SS, Cheng J, et al. RLIP76 is the major ATPdependent transporter of glutathione-conjugates and doxorubicin in human erythrocytes. Arch Biochem Biophys. 2001;391(2):171-179.

54. LaBelle EF, Singh SV, Srivastava SK, Awasthi YC. Dinitrophenyl glutathione efflux from human erythrocytes is primary active ATP-dependent transport. Biochem J. 1986;238(2):443-449.

55. Awasthi YC, Misra G, Rassin DK, Srivastava SK. Detoxification of xenobiotics by glutathione S-transferases in erythrocytes: the transport of the conjugate of glutathione and 1-chloro-2,4-dinitrobenzene. $\mathrm{Br} J$ Haematol. 1983;55(3):419-425.

56. Singhal SS, Wickramarachchi D, Singhal J, Yadav S, Awasthi YC, Awasthi S. Determinants of differential doxorubicin sensitivity between SCLC and NSCLC. FEBS Lett. 2006;580(9):2258-2264.

57. Awasthi S, Singhal SS, Pandya U, et al. ATP-Dependent colchicine transport by human erythrocyte glutathione conjugate transporter. Toxicol Appl Pharmacol. 1999;155(3):215-226.

58.Awasthi YC, Sharma R, Yadav S, Dwivedi S, Sharma A, Awasthi S. The non-ABC drug transporter RLIP76 (RALBP-1) plays a major role in the mechanisms of drug resistance. Curr Drug Metab. 2007;8(4): 315-323.

59. Saxena M, Singhal SS, Awasthi S, et al. Dinitrophenyl S-glutathione ATPase purified from human muscle catalyzes ATP hydrolysis in the presence of leukotrienes. Arch Biochem Biophys. 1992;298(1): 231-237.

60. Sharma R, Singhal SS, Wickramarachchi D, Awasthi YC, Awasthi S. RLIP76 (RALBP1)-mediated transport of leukotriene C4 (LTC4) in cancer cells: implications in drug resistance. Int J Cancer. 2004;112(6): 934-942.

61. Singhal SS, Sharma R, Gupta S, et al. The anionic conjugates of bilirubin and bile acids stimulate ATP hydrolysis by S-(dinitrophenyl)glutathione ATPase of human erythrocyte. FEBS Lett. 1991;281(1-2):255-257.

62. Awasthi S, Sharma R, Singhal SS, Zimniak P, Awasthi YC. RLIP76, a novel transporter catalyzing ATP-dependent efflux of xenobiotics. Drug Metab Dispos. 2002;30(12):1300-1310.

63. Singhal SS, Yadav S, Drake K, Singhal J, Awasthi S. Hsf-1 and POB1 induce drug sensitivity and apoptosis by inhibiting Ralbp1. J Biol Chem. 2008;283(28):19714-19729.

64. Awasthi S, Singhal SS, Yadav S, et al. RLIP76 is a major determinant of radiation sensitivity. Cancer Res. 2005;65(14):6022-6028.

65. Singhal SS, Yadav S, Roth C, Singhal J. RLIP76: A novel glutathioneconjugate and multi-drug transporter. Biochem Pharmacol. 2009;77(5):761-769.
66. Singhal SS, Yadav S, Singhal J, Zajac E, Awasthi YC, Awasthi S Depletion of RLIP76 sensitizes lung cancer cells to doxorubicin. Biochem Pharmacol. 2005;70(3):481-488.

67. Singhal SS, Singhal J, Yadav S, Sahu M, Awasthi YC, Awasthi S. RLIP76: a target for kidney cancer therapy. Cancer Res. 2009;69(10): 4244-4251.

68. Margutti P, Matarrese P, Conti F, et al. Autoantibodies to the C-terminal subunit of RLIP76 induce oxidative stress and endothelial cell apoptosis in immune-mediated vascular diseases and atherosclerosis. Blood. 2008;111(9):4559-4570.

69. Lee S, Wurtzel JG, Singhal SS, Awasthi S, Goldfinger LE. RALBP1/ RLIP76 depletion in mice suppresses tumor growth by inhibiting tumor neovascularization. Cancer Res. 2012;72(20):5165-5173.

70. Folkman J. The role of angiogenesis in tumor growth. Semin Cancer Biol. 1992;3(2):65-71.

71. Heitzer T, Schlinzig T, Krohn K, Meinertz T, Münzel T. Endothelial dysfunction, oxidative stress, and risk of cardiovascular events in patients with coronary artery disease. Circulation. 2001;104(22): 2673-2678.

72. Matarrese P, Colasanti T, Ascione B, et al. Gender disparity in susceptibility to oxidative stress and apoptosis induced by autoantibodies specific to RLIP76 in vascular cells. Antioxid Redox Signal. 2011;15(11): $2825-2836$

73. Komatsu M, Ruoslahti E. R-Ras is a global regulator of vascular regeneration that suppresses intimal hyperplasia and tumor angiogenesis. Nat Med. 2005;11(12):1346-1350.

74. Inuzuka T, Tsuda M, Kawaguchi H, Ohba Y. Transcription factor 8 activates R-Ras to regulate angiogenesis. Biochem Biophys Res Commun 2009;379(2):510-513

75. Sawada J, Komatsu M. Normalization of tumor vasculature by R-Ras. Cell Cycle. 2012;11(23):4285-4286.

76. Sawada J, Urakami T, Li F, et al. Small GTPase R-Ras regulates integrity and functionality of tumor blood vessels. Cancer Cell. 2012;22(2):235-249.

77. Singhal SS, Singhal J, Sharma R, et al. Role of RLIP76 in lung cancer doxorubicin resistance: I. The ATPase activity of RLIP76 correlates with doxorubicin and 4-hydroxynonenal resistance in lung cancer cells. Int J Oncol. 2003;22(2):365-375.

78. Awasthi S, Singhal SS, Singhal J, Cheng J, Zimniak P, Awasthi YC. Role of RLIP76 in lung cancer doxorubicin resistance: II. Doxorubicin transport in lung cancer by RLIP76. Int J Oncol. 2003;22(4): 713-720.

79. Male H, Patel V, Jacob MA, et al. Inhibition of RalA signaling pathway in treatment of non-small cell lung cancer. Lung Cancer. 2012; 77(2):252-259.

80. Awasthi S, Singhal SS, Singhal J, Yang Y, Zimniak P, Awasthi YC. Role of RLIP76 in lung cancer doxorubicin resistance: III. Anti-RLIP76 antibodies trigger apoptosis in lung cancer cells and synergistically increase doxorubicin cytotoxicity. Int J Oncol. 2003;22(4):721-732.

81. Yadav S, Zajac E, Singhal SS, et al. POB1 over-expression inhibits RLIP76-mediated transport of glutathione-conjugates, drugs and promotes apoptosis. Biochem Biophys Res Commun. 2005;328(4):1003-1009.

82. Singhal J, Nagaprashantha L, Vatsyayan R, Awasthi S, Singhal SS. RLIP76, a glutathione-conjugate transporter, plays a major role in the pathogenesis of metabolic syndrome. PLoS One. 2011;6(9):e24688.

mechanisms of action; Optimal clinical use of existing anticancer agents, including combination therapies; Radiation and surgery; Palliative care; Patient adherence, quality of life, satisfaction; Health economic evaluations. The manuscript management system is completely online and includes a very quick and fair peer-review ment sys. Visit http://www. system. Visit http://www.dovepress.com/testimonials.php to read real quotes from published authors.

\section{Dovepress} journal focusing on lung cancer research, identification of therapeutic targets improved outcomes, enhanced survival and quality of life for the cancer patient. Specific topics covered in the journal include: Epidemiology, detection and screening; Cellular research and biomarkers; Identification of biotargets and agents with nove Submit your manuscript here: http://www.dovepress.com/lung-cancer-targets--therapy-journal

\section{Publish your work in this journal}

Lung Cancer:Targets and Therapy 2013:4

submit your manuscript $\mid$ www.dovepress.com 